\title{
Track me to track us: Leveraging short range wireless technologies for enabling energy efficient Wi-Fi-based localization
}

\author{
Mohamed Abdellatif, Abderrahmen Mtibaa, Khaled Harras
}

Carnegie Mellon University in Qatar, Doha, QATAR

m.tarek.abdellatif@gmail.com

Given the success of outdoor tracking via GPS and the rise of real-time context-aware services, users will soon rely on applications that require higher granularity indoor localization. This need is further manifested in countries like Qatar, where various social and business activities occur indoors. Wi-Fi-based indoor localization is one of the most researched techniques due to its ubiquitous deployment and acceptable accuracy for a wide range of applications. However, we do not witness such techniques widely deployed mainly due to their high demand on energy, which is a precious commodity in mobile devices.

We propose an energy-efficient indoor localization system that leverages peoples' typical group mobility patterns and short-range wireless technologies available on their devices. Our system architecture, shown in the figure, is designed to be easily integrated with existing Wi-Fi localization engines. We first utilize low-energy wireless technologies, such as Bluetooth, to detect and cluster individuals moving together. Our system then assigns a group representative to act as a designated cluster head that would be constantly tracked. The location of other group members can be inferred so long as they remain within proximity of the cluster heads. Afterwards, cluster heads continue to send the periodic received signal strength indicator (RSSI) updates, while the remaining members turn off their Wi-Fi interface relying on the cluster head to be localized. Our system is responsible for dynamically handling the merger or splitting of clusters as a result of mobility.

We implement a prototype of the system, and evaluate it at scale using the QualNet simulator. Our results show that we can achieve up to 55\% energy reduction with a relatively small degradation in localization accuracy averaging 2 meters. This accuracy reduction is non-impactful given the typical applications expected to leverage our system. 